\title{
GUIDELINES
}

\section{Chapter 8. CKD and lifestyle-related diseases}

(C) Japanese Society of Nephrology 2009

- An unhealthy lifestyle, such as obesity, insufficient exercise, alcohol, smoking, and other stresses, are assumed to be implicated in the development of CKD.

- Improvement in lifestyle has proven valuable in managing/treating CKD development and progression.

- Lifestyle-related diseases and metabolic syndrome have become popular subjects (Table 8-1). A lifestyle-related disease is defined as "a disorder whose development is greatly affected by individual lifestyle habits as well as genetic background". Metabolic syndrome is a concept that excessive eating and lack of exercise causes fat accumulation in visceral organs, resulting in hypertension, diabetes, and dyslipidemia. Insulin resistance is considered to be an underlying causal factor in metabolic syndrome.
- Lifestyle-related disease and metabolic syndrome are closely related to the development of CKD. Obesity, particularly abdominal obesity that accumulates visceral fat, can easily cause proteinuria or reduced kidney function. An epidemiologic study conducted in Japan has reported that patients with metabolic syndrome had a higher cumulative incidence and relative risk of $\mathrm{CKD}$ (Fig. 8-1).

- The prevalence of metabolic syndrome is currently increasing among the Japanese general population. Kidney dysfunction due to obesity is implied by insulin resistance, the magnitude of which has a positive relationship with the degree of proteinuria. Insulin resistance increases with decreasing in kidney function, thus producing vicious cycle. A similar vicious cycle arises in CKD between risk factors, such as high blood pressure and dyslipidemia (Fig. 8-2). It has recently been acknowledged that high blood pressure or obesity without diabetes also causes kidney dysfunction.

Table 8-1 Criteria of metabolic syndrome

\begin{tabular}{ll}
\hline $\begin{array}{l}\text { Storage of visceral fat (visceral adiopocytes) } \\
\text { Waist circumference }\end{array}$ & $\begin{array}{l}\text { Men } \geq 85 \mathrm{~cm} \\
\text { Women } \geq 90 \mathrm{~cm}\end{array}$ \\
& Area of visceral fat: $\mathrm{men} / \mathrm{women} \geq 100 \mathrm{~cm}^{2}$ \\
Above and following factor of 2 and over & $\geq 50 \mathrm{mg} / \mathrm{dL} \mathrm{and} / \mathrm{or}<40 \mathrm{mg} / \mathrm{dL}$ \\
Hypertriglyceridemia and/or low high-density lipoprotein cholesterol & $\geq 130 \mathrm{mmHg}$ and/or $\geq 85 \mathrm{mmHg}$ \\
Systolic blood pressure and/or diastolic blood pressure & $\geq 110 \mathrm{mg} / \mathrm{dL}$ \\
Fasting blood glucose &
\end{tabular}

Data were obtained, with modification, from the J Jpn Soc Int Med 2005;94:794-809 (in Japanese) 
Fig. 8-1 Incidence (left panel) and relative risk (right panel) of developing chronic kidney disease $(C K D)$ in the presence $(+) /$ absence $(-)$ of metabolic syndrome $(M S)$. GFR

Glomerular filtration rate, $D M$ diabetes mellitus. The data are quoted, with modification, from Ninomiya $\mathrm{T}$ et al. (Am J Kidney Dis 2006;48:383-391)
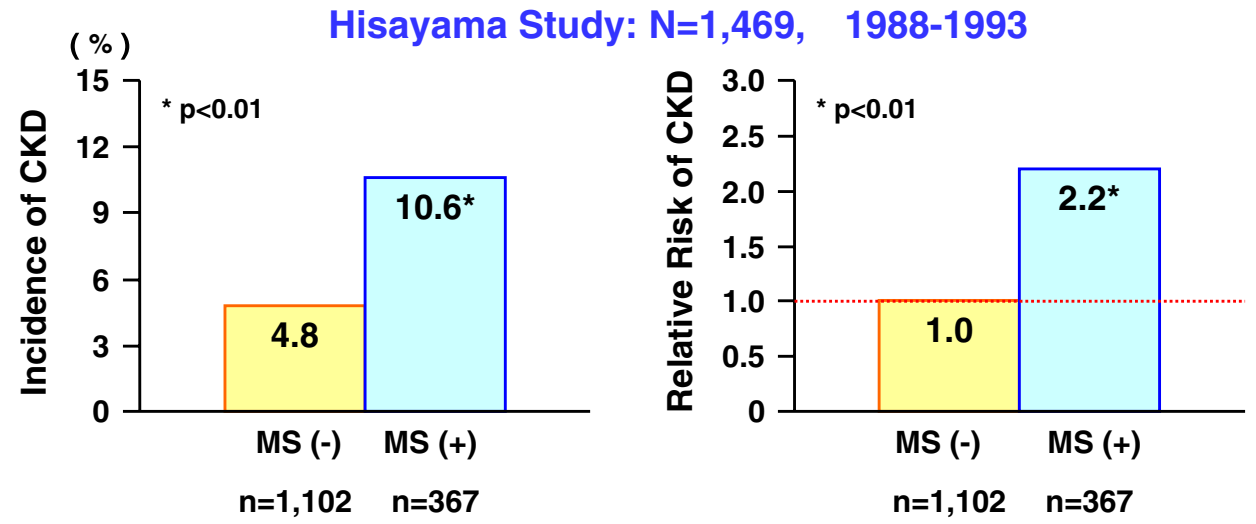

Cumulative incidence was age and sex adjusted.

Relative risk was adjusted for age, sex, GFR, proteinuria, serum albumin, total cholesterol, hemoglobin, smoking, and drinking.
Fig. 8-2 Lifestylerelated visceral obesity and its relationship with CKD and other associated medical conditions. ASO Atherosclerotic disease

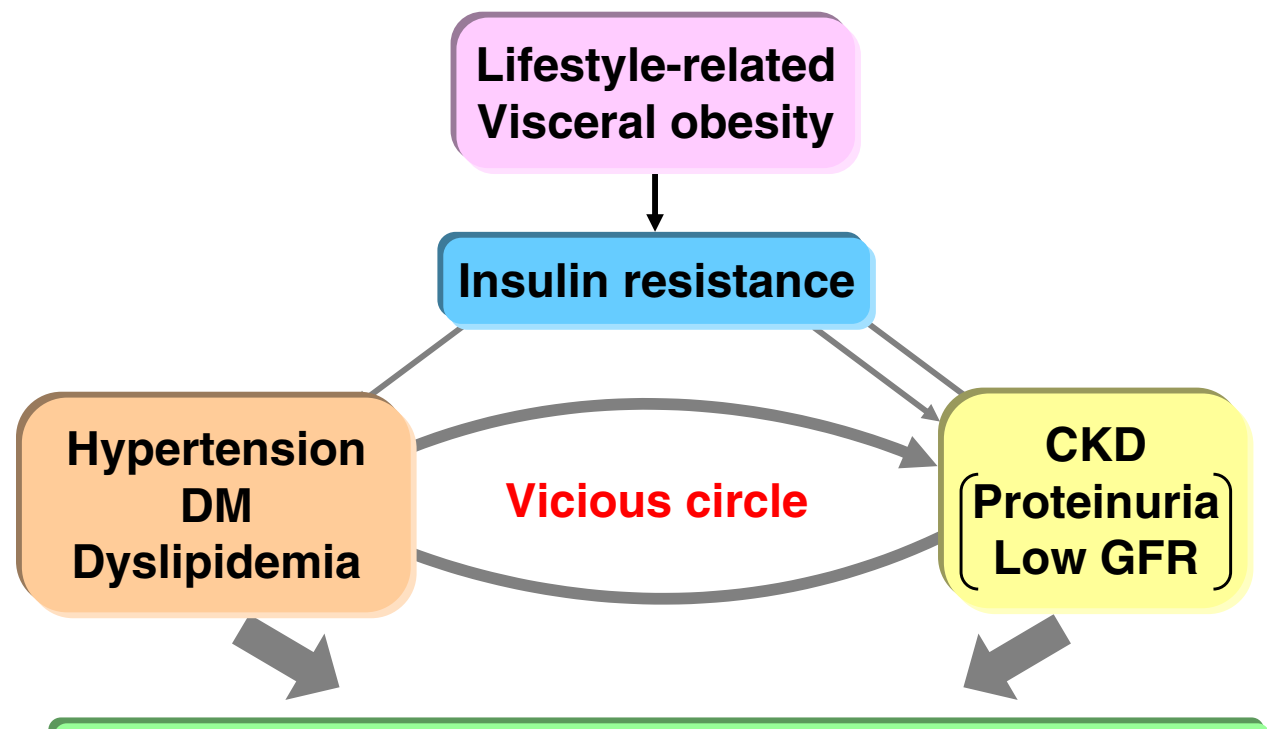

Stroke, Acute myocardial infarction, Congestive heart failure, End-stage renal disease, ASO 